\title{
Umwandlungssteuerrecht
}




\section{Gernot Brähler}

\section{Umwandlungssteuerrecht}

\section{Grundlagen für Studium und Steuerberaterprüfung}

8., aktualisierte Auflage

Unter Mitarbeit von:

Dipl.-Kfm. Markus Bensmann

Dipl.-Kfm. Dr. Andreas Krenzin, StB

Springer Gabler 
Prof. Dr. Gernot Brähler

TU Ilmenau, Deutschland

ISBN 978-3-658-03882-3

ISBN 978-3-658-03883-0 (eBook)

DOI 10.1007/978-3-658-03883-0

Die Deutsche Nationalbibliothek verzeichnet diese Publikation in der Deutschen Nationalbibliografie; detaillierte bibliografische Daten sind im Internet über http://dnb.d-nb.de abrufbar.

Springer Gabler

(c) Springer Fachmedien Wiesbaden 2004, 2008, 2009, 2011, 2013, 2014

Das Werk einschließlich aller seiner Teile ist urheberrechtlich geschützt. Jede Verwertung, die nicht ausdrücklich vom Urheberrechtsgesetz zugelassen ist, bedarf der vorherigen Zustimmung des Verlags. Das gilt insbesondere für Vervielfältigungen, Bearbeitungen, Übersetzungen, Mikroverfilmungen und die Einspeicherung und Verarbeitung in elektronischen Systemen.

Die Wiedergabe von Gebrauchsnamen, Handelsnamen, Warenbezeichnungen usw. in diesem Werk berechtigt auch ohne besondere Kennzeichnung nicht zu der Annahme, dass solche Namen im Sinne der Warenzeichen- und Markenschutz-Gesetzgebung als frei zu betrachten wären und daher von jedermann benutzt werden dürften.

Lektorat: Irene Buttkus

Gedruckt auf säurefreiem und chlorfrei gebleichtem Papier

Springer Gabler ist eine Marke von Springer DE. Springer DE ist Teil der Fachverlagsgruppe Springer Science+Business Media.

www.springer-gabler.de 


\section{Vorwort zur 8. Auflage}

Das Umwandlungssteuerrecht stellt eine äußerst komplexe und vielschichtige Materie dar, da neben dem eigentlichen UmwStG immer wieder Bezug auf Regelungsbereiche etwa des EStG, des KStG sowie des Bilanzrechts genommen wird. Sind allerdings die grundlegenden steuerlichen Problembereiche und Regelungszusammenhänge verstanden worden, zeigen sich die Vorschriften des UmwStG vielfach als lediglich konsequente Umsetzung dieser steuerlichen Grundsätze. Im Rahmen dieses Lehrbuchs werden daher einleitend stets die relevanten steuerlichen Grundprinzipien erklärt, so dass die Regelungen des UmwStG als notwendige und nachvollziehbare Folge dieser Grundprinzipien erscheinen. Auf diese Weise zeigt sich das Umwandlungssteuerrecht als überschaubares und verständliches System, das - zumindest in weiten Teilen - in sich schlüssig ist. Die Erläuterung der steuerlichen Grundprinzipien erlaubt aber auch hierbei, die bestehenden Inkonsistenzen der Gesetzgebung aufzuzeigen und kritisch zu hinterfragen. In der vorliegenden achten Auflage werden unter anderem auch die aktuellen Änderungen des Umwandlungssteuergesetzes durch das Amtshilferichtlinie-Umsetzungsgesetz vom 26.06.2013 berücksichtigt. Der Gesetzgeber hat hier insbesondere die Verlustverrechnungsbeschränkung durch eine Erweiterung des steuerlichen Rückwirkungsbegriffs auf den übernehmenden Rechtsträger erweitert.

Angesichts der hohen Komplexität ist es das Ziel dieses Lehrbuchs, die Grundzüge und Hauptproblemstellungen einfach und strukturiert darzustellen. Aus diesem Grunde wurden zahlreiche Beispiele, Abbildungen und Übersichten verwendet, die zu einer besseren Veranschaulichung der Problemfelder beitragen sollen. Auch dienen die Merkfelder sowie die Zusammenfassungen einer zielgerichteten Vorbereitung auf Examens- und/oder Steuerberaterprüfungen.

Das Buch basiert auf dem Rechtsstand 01. September 2013.

Gernot Brähler 


\section{Inhaltsverzeichnis}

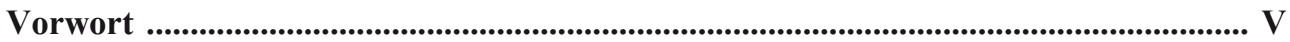

Inhaltsverzeichnis ............................................................................................................. VII

Abbildungsverzeichnis ..................................................................................................... XIX

Abkürzungs- und Symbolverzeichnis ........................................................................ XXVII

\section{KAPITEL I: ÜBERBLICK ZUM UMWANDLUNGSRECHT}

$1 \quad$ Einleitung..............................................................................................................................

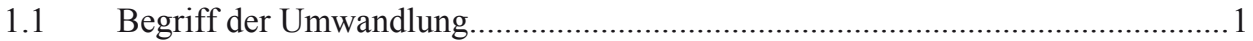

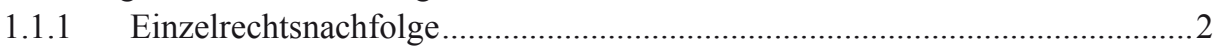

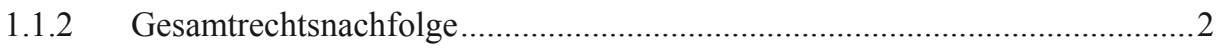

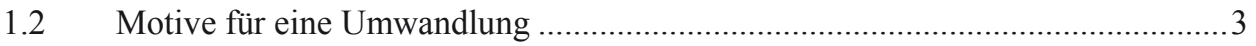

2 Rechtsformneutralität der Besteuerung..........................................................................6

$2.1 \quad$ Besteuerung von Kapitalgesellschaften...........................................................6

2.2 Besteuerung von Einzelunternehmen und Personengesellschaften .......................9

2.3 Vergleich der Steuerbelastung zwischen KapGes und EU/PersGes......................14

3 Umwandlungsgesetz, Umwandlungssteuergesetz und

Umwandlungssteuererlass..............................................................................................17

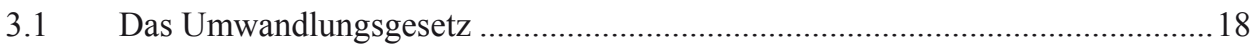

3.1.1 Verschmelzung nach dem Umwandlungsgesetz.........................................22

3.1.2 Spaltung nach dem Umwandlungsgesetz..................................................22

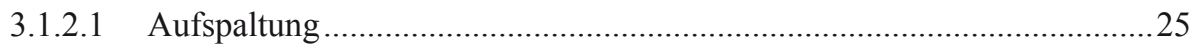

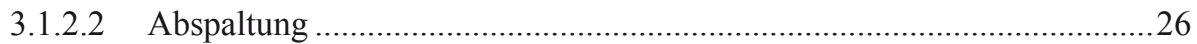

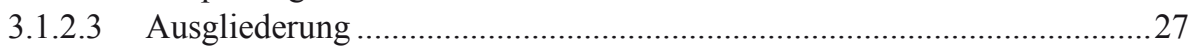

3.1.3 Vermögensübertragung nach dem Umwandlungsgesetz ……………….......28

3.1.4 Formwechsel nach dem Umwandlungsgesetz ……….................................28

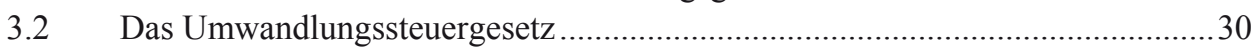

3.2.1 Aufbau des Umwandlungssteuergesetzes ..................................................... 31

3.2.2 Beziehung zwischen Umwandlungsgesetz und

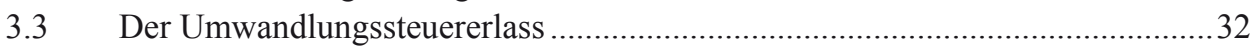




\section{KAPITEL II: VERSCHMELZUNG VON KAPITALGESELLSCHAFTEN AUF PERSONENGESELLSCHAFTEN}

1 Allgemeines

2 Verschmelzungsmotive .34

3 Handelsrechtliche Regelungen ....................................................................................35

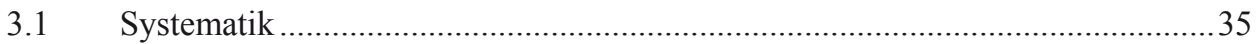

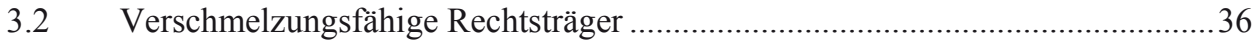

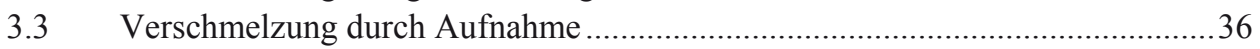

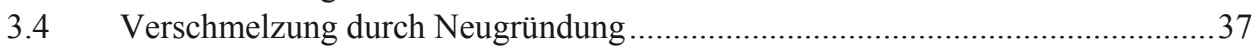

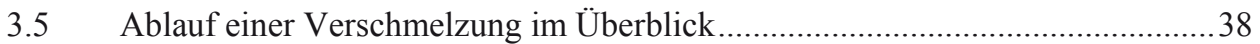

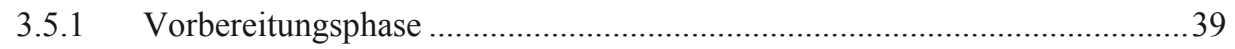

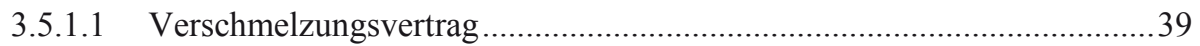

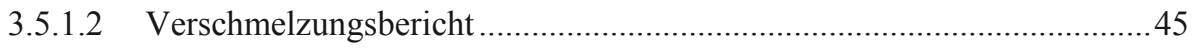

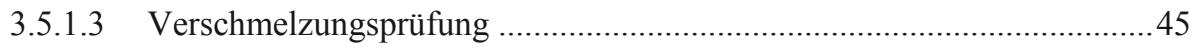

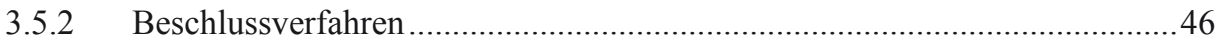

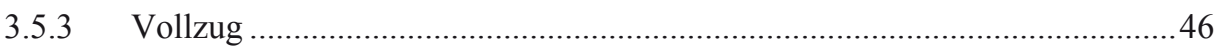

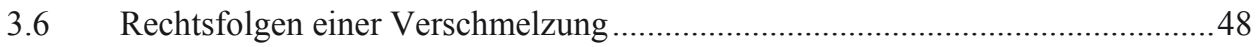

3.7 Grenzüberschreitende Verschmelzung ..........................................................48

4 Bilanzielle Behandlung in der Handelsbilanz ......................................................49

4.1 Bilanzierung bei der übertragenden Kapitalgesellschaft .................................50

4.2 Bilanzierung bei der übernehmenden Personengesellschaft.............................50

5 Steuerrechtliche Regelungen .......................................................................55

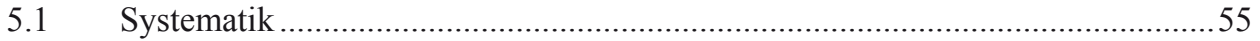

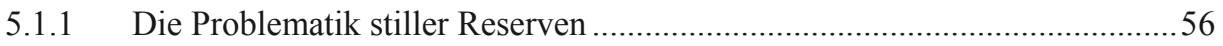

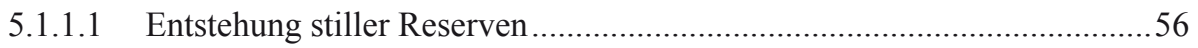

5.1.1.2 Realisation und Besteuerung von stillen Reserven ................................56

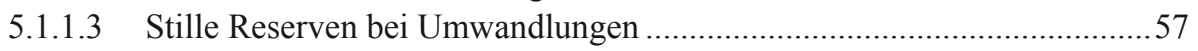

5.1.2 Die Problematik der Gewinnrücklagen..........................................................61

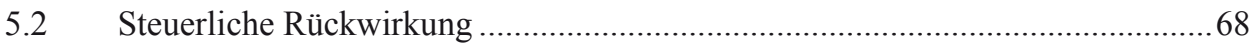

5.2.1 Handelsrechtlicher Umwandlungsstichtag und steuerlicher

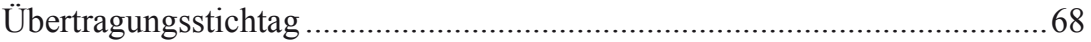

5.2.2 Steuerlicher Rückwirkungszeitraum ...................................................... 70

5.2.3 Probleme im steuerlichen Rückwirkungszeitraum ....................................... 71

5.2.3.1 Ausscheiden von Anteilseignern ..................................................... 71

5.2.3.2 Gewinnausschüttungen der übertragenden Kapitalgesellschaft .................72

5.2.3.3 Zahlungen an Gesellschafter der übertragenden Kapitalgesellschaft........76 
5.2.3.4 Lieferungen und Leistungen zwischen übertragender Kapitalgesellschaft und übernehmender Personengesellschaft .................77

5.2.3.5 Pensionszusagen an Gesellschafter der übertragenden

Kapitalgesellschaft .78

5.2.4 Grenzüberschreitende Umwandlungen und steuerlicher

Rückwirkungszeitraum .79

5.2.5 Einschränkung der Verlustverrechnung mit einem Übertragungsgewinn bei Umwandlungen im Rückwirkungszeitraum..... .80

5.3 Auswirkungen bei der übertragenden Kapitalgesellschaft ................................83

5.3.1 Wertansatz in der steuerlichen Schlussbilanz ............................................. 83

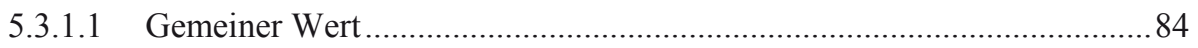

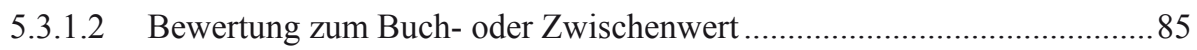

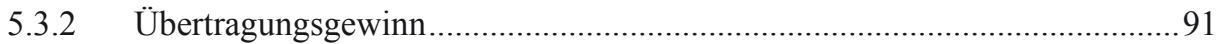

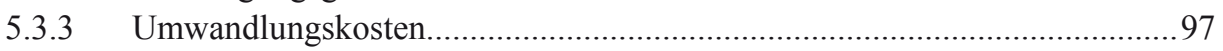

5.3.4 Mögliche Vorteilhaftigkeit eines Zwischenwertansatzes...............................98

5.3.5 Verschmelzungen mit ausländischer Beteiligung .................................... 101

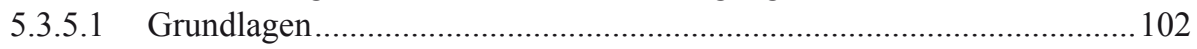

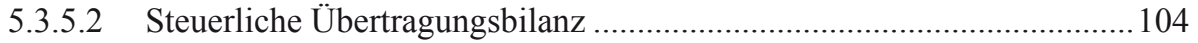

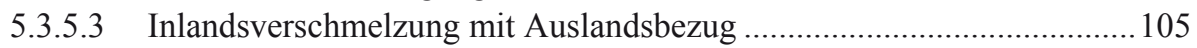

5.3.5.4 Herausverschmelzung .................................................................. 113

5.3.5.5 Auslandsverschmelzung mit Inlandsbezug und Hineinverschmelzung... 116

5.4 Auswirkungen bei der übernehmenden Personengesellschaft......................... 118

5.4.1 Wertverknüpfung und Wertaufholung........................................................ 118

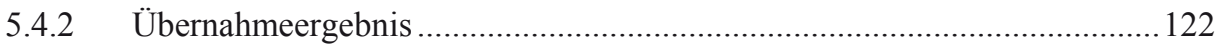

5.4.2.1 Ausschüttungsfiktion der offenen Rücklagen ....................................... 122

5.4.2.2 Ermittlung des Übernahmeergebnisses ................................................ 125

5.4.2.2.1 Anschaffungs- und Einlagefiktion ( $(5 \mathrm{UmwStG})$.......................... 134

5.4.2.2.2 Ermittlung des Übernahmeergebnisses unter Berücksichtigung der Einlagefiktion ...................................................................... 140

5.4.2.2.3 Umbewertungen nach $\S 4$ Abs. 4 Satz 2 UmwStG.......................... 143

5.4.2.3 Besteuerung des Übernahmeergebnisses.............................................. 149

5.4.2.4 Zusammenfassung und Zusammenhang zwischen Übertragungs- und

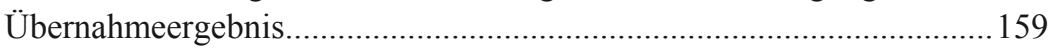

5.4.2.5 Bilanzielle Behandlung des Übernahmeergebnisses ............................... 163

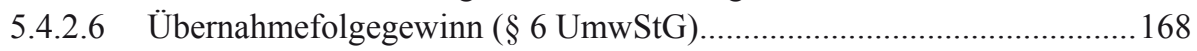

5.4.3 Eintritt in die Rechtsposition der übertragenden Kapitalgesellschaft .......... 170

5.4.3.1 Abschreibung der übernommenen Wirtschaftsgüter und

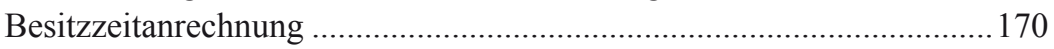

5.4.3.2 Steuerliche Behandlung von Verlustvorträgen........................................ 174

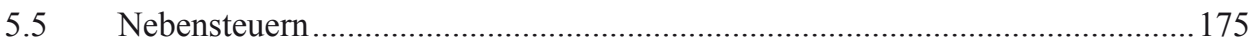

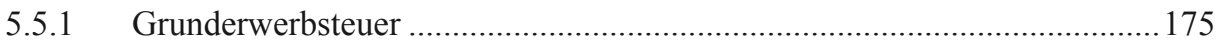

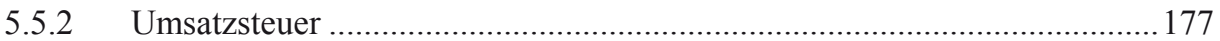


6 Umwandlung einer KapGes in eine Einzelunternehmung durch

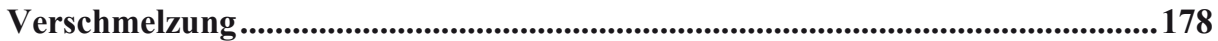

7 Umwandlung einer KapGes in eine PersGes durch Formwechsel........................180

7.1 Zivil- und handelsrechtliche Regelungen ....................................................... 180

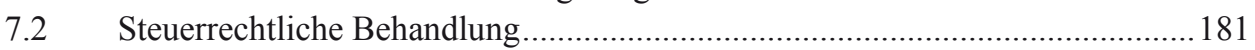

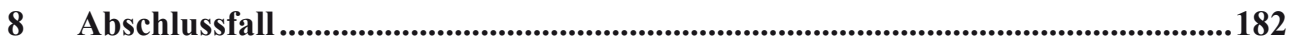

KAPITEL III: VERSCHMELZUNG VON KAPITALGESELLSCHAFTEN

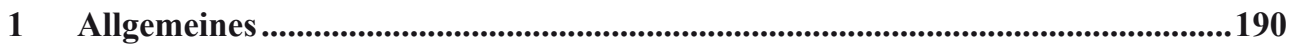

2 Verschmelzungsmotive .................................................................................................194

3 Handelsrechtliche Regelungen ............................................................................................. 195

3.1 Verschmelzung von Kapitalgesellschaften im UmwG .................................. 195

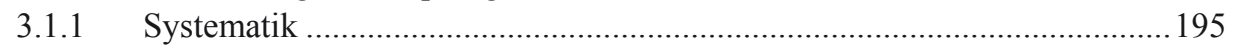

3.1.2 Verschmelzungsfähige Kapitalgesellschaften ............................................195

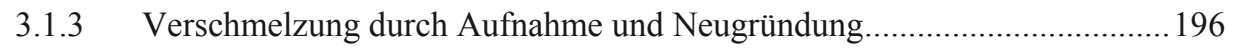

3.2 Ablauf der Verschmelzung im Überblick........................................................ 197

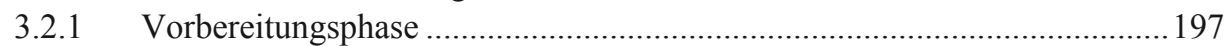

3.2.1.1 Vertragliche Grundlagen der Verschmelzung ....................................... 197

3.2.1.1.1 Nationale Verschmelzungen - Verschmelzungsvertrag .................... 197

3.2.1.1.2 Grenzüberschreitende Verschmelzung - Verschmelzungsplan........ 198

3.2.1.2 Umtauschverhältnis und bare Zuzahlungen ........................................200

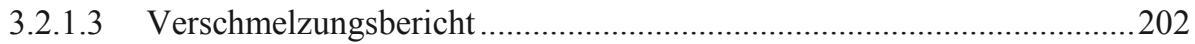

3.2.1.4 Verschmelzungsprüfung und Prüfungsbericht ......................................203

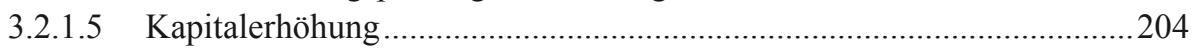

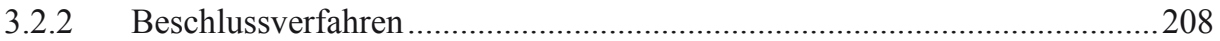

3.2.3 Schutz der Minderheitsgesellschafter und Gläubiger .................................208

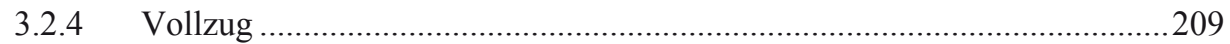

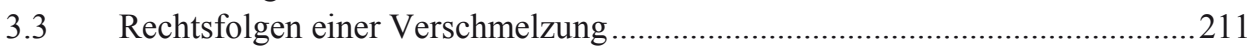

4 Bilanzielle Behandlung in der Handelsbilanz ..................................................211

4.1 Bilanzierung bei der übertragenden Kapitalgesellschaft .................................211

4.2 Bilanzierung bei der übernehmenden Kapitalgesellschaft...............................211

5 Steuerrechtliche Regelungen ........................................................................212 


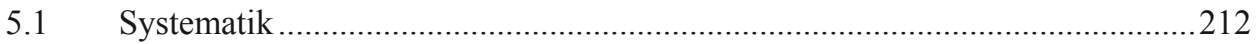

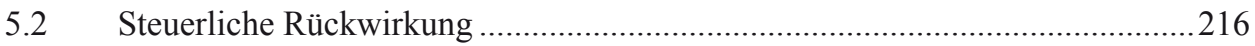

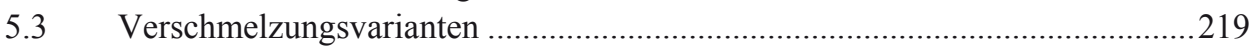

5.3.1 Verschmelzung von Kapitalgesellschaften ohne gegenseitige

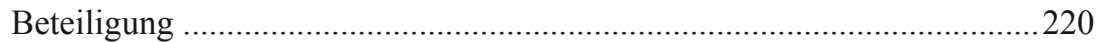

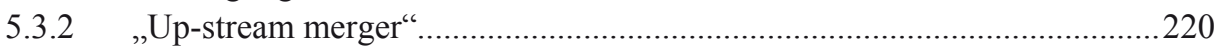

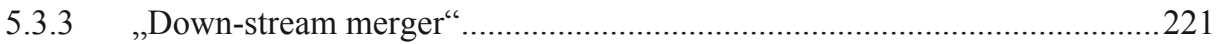

5.3.4 „Up-stream merger“ und „down-stream merger“" im Vergleich ...................2223

5.3.5 Kombinierter ,up-stream/ down-stream merger“...........................................225

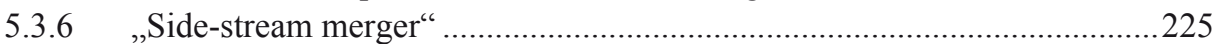

5.4 Auswirkungen bei der übertragenden Kapitalgesellschaft .................................226

5.4.1 Bewertungswahlrecht gem. § 11 Abs. 2 UmwStG .....................................226

5.4.1.1 Sicherstellung der späteren Besteuerung i.S.d. § 11 Abs. 2 Satz 1

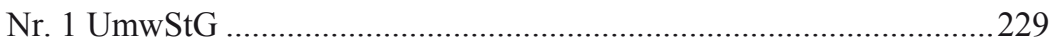

5.4.1.2 Ausschluss oder Beschränkung des deutschen Besteuerungsrechts i.S.d. § 11 Abs. 2 Satz 1 Nr. 2 UmwStG ...............................................231

5.4.1.3 Gegenleistung i.S.d. § 11 Abs. 2 Satz 1 Nr. 3 UmwStG ..........................240

5.4.2 Konsequenzen bei fehlenden Voraussetzungen des § 11 Abs. 2 Satz 1

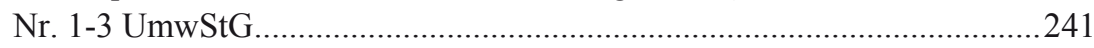

5.4.3 Sonderfall: Anrechnung einer fiktiven ausländischen Steuer ........................243

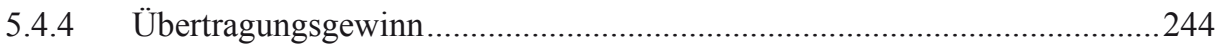

5.4.5 Wertaufholungsgebot bei down-stream merger........................................247

5.5 Auswirkungen bei der übernehmenden Kapitalgesellschaft..............................250

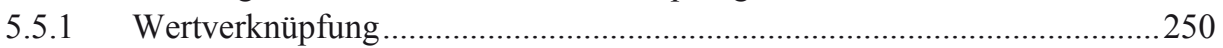

5.5.2 Übernahmeergebnis der übernehmenden Kapitalgesellschaft ......................252

5.5.2.1 Entstehung des Übernahmeergebnisses...................................................252

5.5.2.2 Besteuerung des Übernahmeergebnisses.................................................258

5.5.3 Übernahmeergebnis in Fällen, in denen kein (100 \%iger) „up-stream

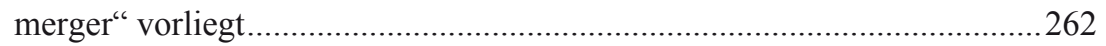

5.5.4 Wertaufholungsgebot bei up-stream merger................................................2263

5.5.5 Eintritt in die Rechtsposition der übertragenden Kapitalgesellschaft ..........266

5.5.6 Behandlung von Verlustvorträgen .............................................................2267

5.5.6.1 Fortführung eines Verlustvortrags der Übernehmerin..............................267

5.5.6.2 Nutzung eines Verlustvortrags der Überträgerin.....................................2277

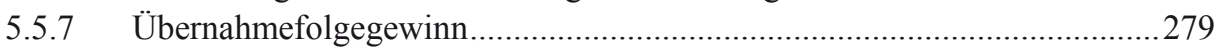

5.6 Auswirkungen auf den steuerlichen Eigenkapitalausweis.................................281

5.6.1 Behandlung bei Umwandlungen gem. § $29 \mathrm{KStG}$.......................................283

5.6.1.1 Exkurs: Kapitalerhöhung und -herabsetzung gem. § $28 \mathrm{KStG}$.................285

5.6.1.1.1 Kapitalerhöhung aus Gesellschaftsmitteln ......................................28

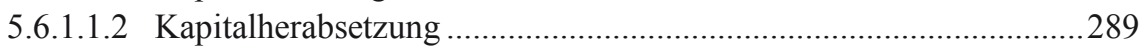

5.6.1.1.3 Minderung des Sonderausweises durch das steuerliche Einlagekonto

5.6.1.2 Fiktion der Herabsetzung des Nennkapitals der übertragenden Kapitalgesellschaft gem. § 29 Abs. 1 KStG 
5.6.1.3 Übergang des Bestands des steuerlichen Einlagekontos .........................295

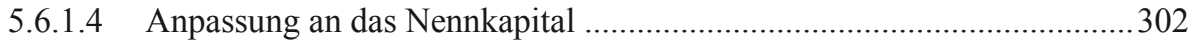

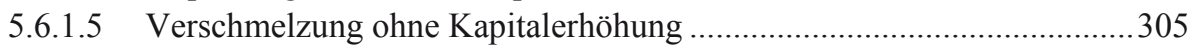

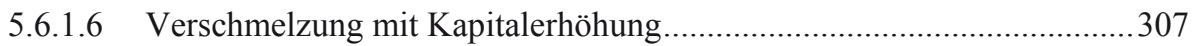

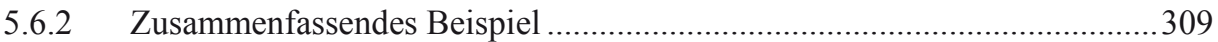

5.7 Steuerliche Folgen für die Gesellschafter der übertragenden

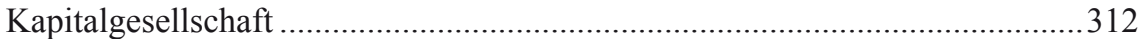

5.7.1 Ausschluss oder Beschränkung des deutschen Besteuerungsrechts i.S.d.

$\S 13$ Abs. 2 Satz 1 Nr. 1 UmwStG ............................................................. 315

5.7.2 Anwendung der Fusionsrichtlinie 90/434/EWG i.S.d. § 12 Abs. 2 Satz 1

Nr. 2 UmwStG ...................................................................................... 320

5.7.3 Steuerliche Qualifikation untergehender und neu gewährter Anteile bei

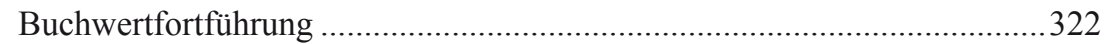

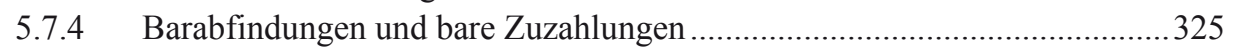

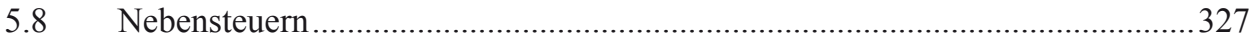

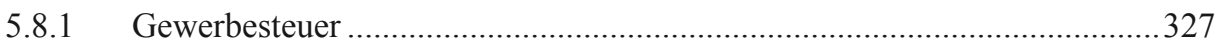

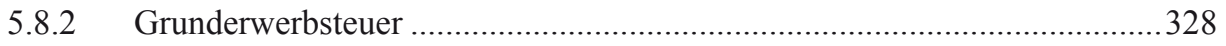

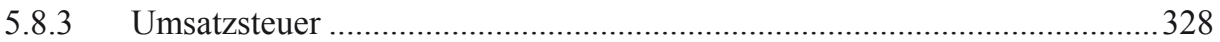

6 Verschmelzung von Kapitalgesellschaften über die Grenze...................................328

6.1 Voraussetzung für die Anwendbarkeit des UmwStG .....................................329

6.2 Konsequenzen grenzüberschreitender Verschmelzungen von

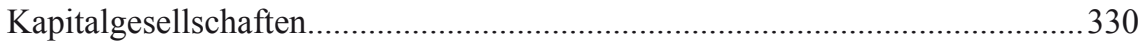

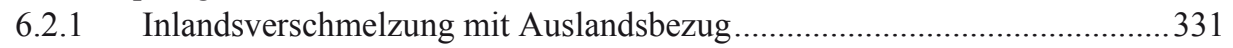

6.2.1.1 Konsequenzen für die übertragende Kapitalgesellschaft........................331

6.2.1.2 Konsequenzen für die übernehmende Kapitalgesellschaft .....................332

6.2.1.3 Konsequenzen für die Anteilseigner der übertragenden KapGes..............332

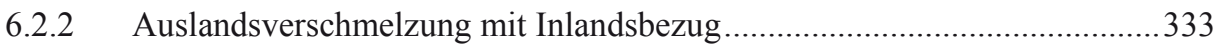

6.2.2.1 Konsequenzen für die übertragende Kapitalgesellschaft..........................333

6.2.2.2 Konsequenzen für die übernehmende Kapitalgesellschaft .......................333

6.2.2.3 Konsequenzen für die Anteilseigner der übertragenden KapGes.............333

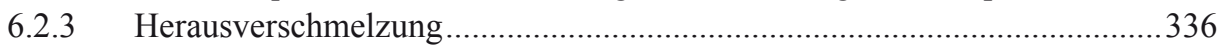

6.2.3.1 Konsequenzen für die übertragende Kapitalgesellschaft........................336

6.2.3.2 Konsequenzen für die übernehmende Kapitalgesellschaft .......................337

6.2.3.3 Konsequenzen für die Anteilseigner der übertragenden KapGes.............337

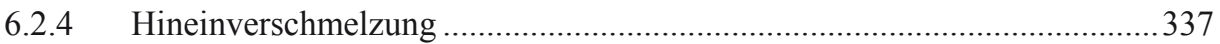

6.2.4.1 Konsequenzen für die übertragende Kapitalgesellschaft..........................337

6.2.4.2 Konsequenzen für die übernehmende Kapitalgesellschaft ......................338

6.2.4.3 Konsequenzen für die Anteilseigner der übertragenden KapGes.............339

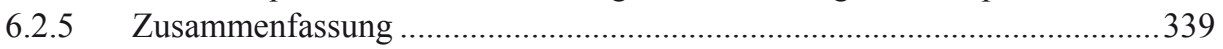

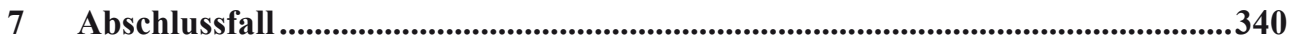




\section{KAPITEL IV： SPALTUNG VON KAPITALGESELLSCHAFTEN}

1 Allgemeines .347

2 Spaltungsmotive .347

3 Handelsrechtliche Regelungen .............................................................................348

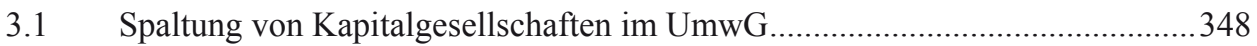

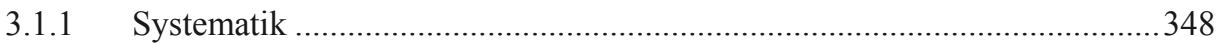

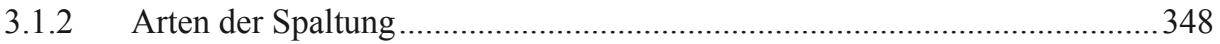

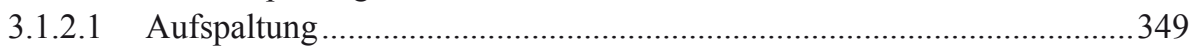

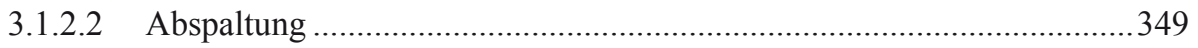

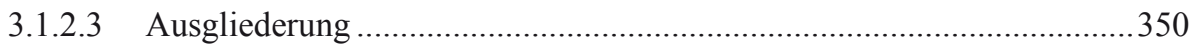

3.1.3 Entsprechende Anwendung der Verschmelzungsvorschriften.....................351

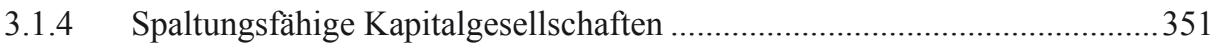

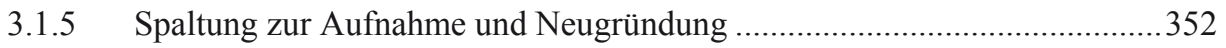

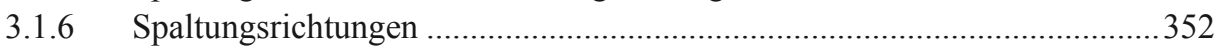

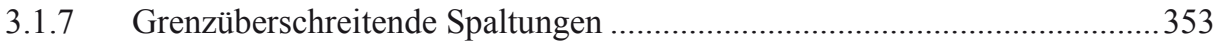

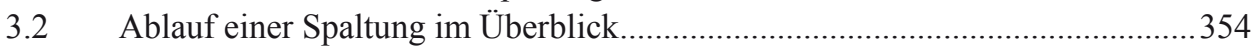

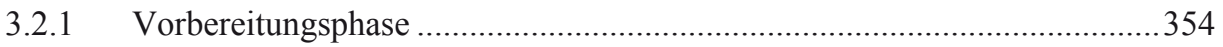

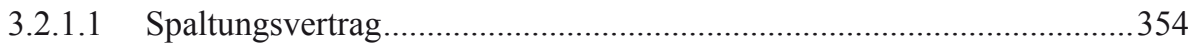

3.2.1.1.1 Umtauschverhältnis und bare Zuzahlungen sowie Einzelheiten

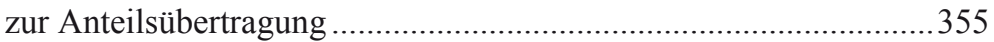

3.2.1.1.2 Aufteilung und Zuordnung des Vermögens .....................................356

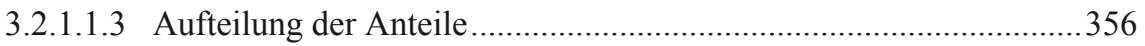

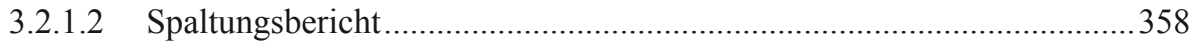

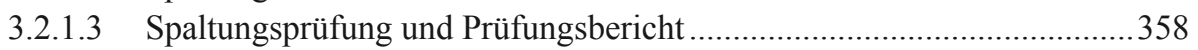

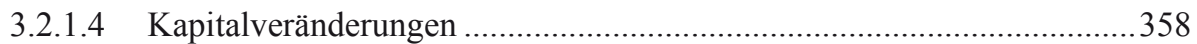

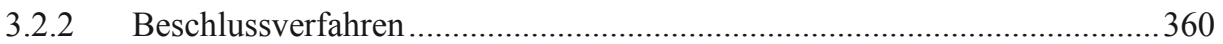

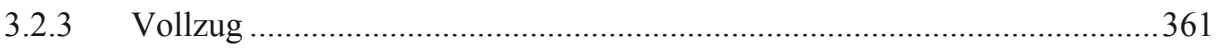

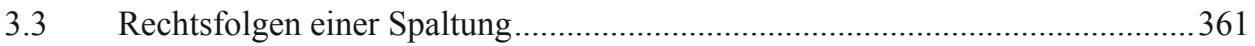

4 Bilanzielle Behandlung in der Handelsbilanz .......................................................362

4.1 Bilanzierung bei der übertragenden Kapitalgesellschaft .................................362

4.2 Bilanzierung bei der oder den übernehmenden Kapitalgesellschaft(en) ............363

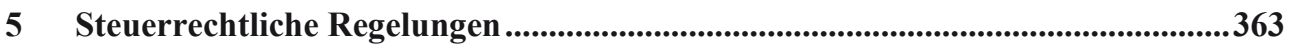

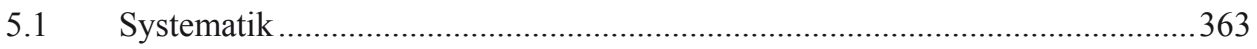

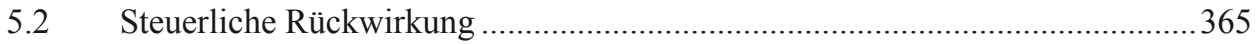

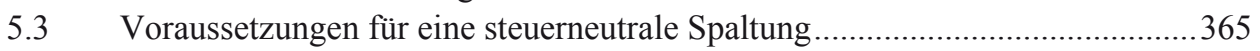

5.3.1 Teilbetriebsvoraussetzung in $\S 15$ Abs. 1 Satz 2 UmwStG ..........................365

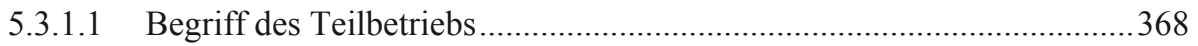


5.3.1.2 Zuordnung der Wirtschaftsgüter zu den Teilbetrieben............................369

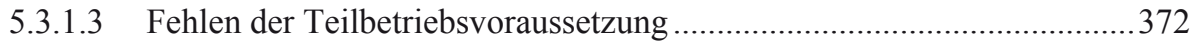

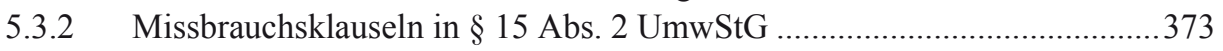

5.3.2.1 Erwerb und Aufstockung von Mitunternehmeranteilen und 100 \%igen Beteiligungen an Kapitalgesellschaften ................................373

5.3.2.2 Veräußerung bzw. Vorbereitung der Veräußerung ...................................376

5.3.2.2.1 Veräußerung an außenstehende Personen ( $§ 15$ Abs. 2 Satz 2 UmwStG) ........................................................................... 379

5.3.2.2.2 Vorbereitung der Veräußerung ( $§ 15$ Abs. 2 Satz 3 und 4 UmwStG) .381

5.3.2.2.3 Rechtsfolgen einer schädlichen Anteilsveräußerung .........................384

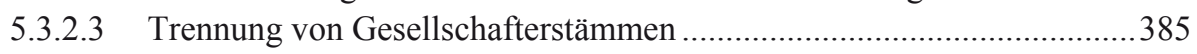

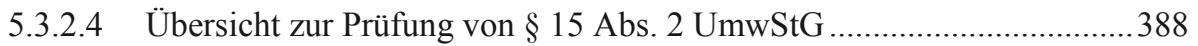

5.4 Auswirkungen bei der zu spaltenden Kapitalgesellschaft .................................389

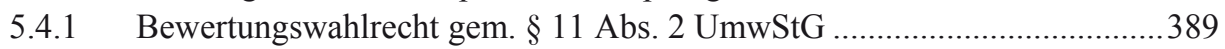

5.4.2 Wertaufholungsgebot gem. § 11 Abs. 2 Satz 2, 3 UmwStG .........................391

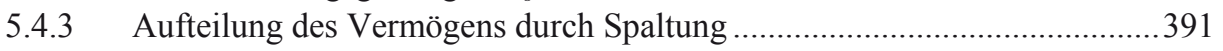

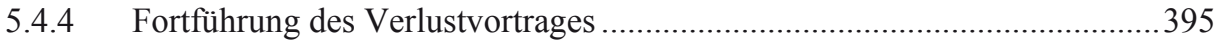

5.4.5 Aufteilung des steuerlichen Eigenkapitalausweises ......................................396

5.5 Auswirkungen bei der übernehmenden Kapitalgesellschaft...............................397

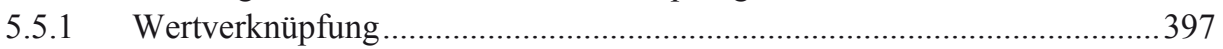

5.5.2 Übernahmeergebnis der übernehmenden Kapitalgesellschaft .......................397

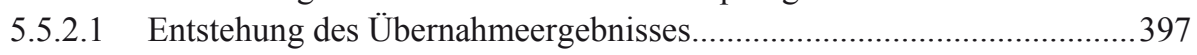

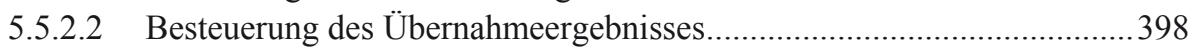

5.5.3 Wertaufholungsgebot gem. $\S 12$ Abs. 1 Satz 2 UmwStG............................403

5.5.4 Eintritt in die Rechtsposition der übertragenden Kapitalgesellschaft ..........404

5.5.5 Behandlung von Verlustvorträgen ..........................................................405

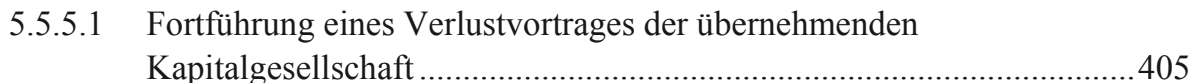

5.5.5.2 Nutzung des Verlustvortrages der spaltenden Kapitalgesellschaft...........405

5.5.6 Übernahmefolgegewinn....................................................................... 408

5.6 Auswirkungen auf den steuerlichen Eigenkapitalausweis.................................408

5.6.1 Kapitalveränderungen bei Spaltungen gem. $\S 29 \mathrm{KStG}$.............................410

5.6.1.1 Fiktion der Herabsetzung des Nennkapitals der zu spaltenden Kapitalgesellschaft gem. § 29 Abs. 1 KStG ..........................................410

5.6.1.2 Aufteilung und Übergang des Bestands des steuerlichen Einlagekontos ..........................................................................4 411

5.6.1.3 Anpassung der Nennkapitalien der beteiligten Kapitalgesellschaften.....416

5.7 Steuerliche Folgen für die Gesellschafter der übertragenden

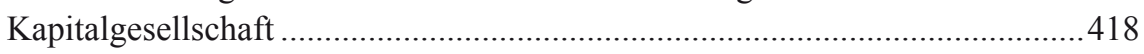

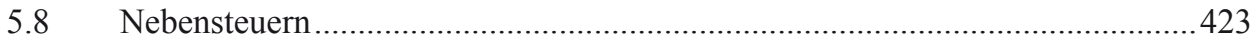

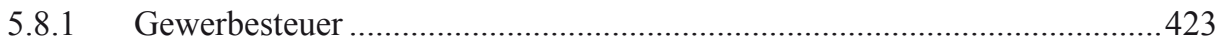

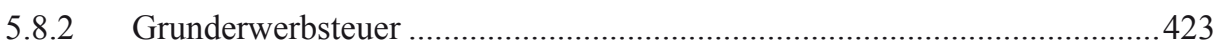

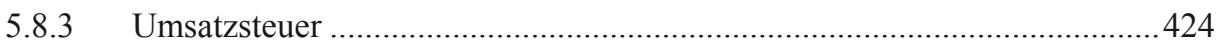


6 Gesamtübersicht. .425

\section{KAPITEL V: EINBRINGUNG IN KAPITAL- UND PERSONENGESELL- SCHAFTEN}

1 Allgemeines .439

1.1 Systematik 439

1.2 Der Einbringungskreislauf 453

2 Einbringung in eine Kapitalgesellschaft gem. $§ 20 \mathrm{UmwStG}$ 455

2.1 Systematik 457

2.2 Einbringungsmotive 459

2.2.1 Konzernumstrukturierung. .459

2.2.2 Wechsel der Rechtsform. 459

2.3 Zivilrechtliche Anwendungsfälle . 459

2.3.1 Gesamtrechtsnachfolge. 460

2.3.2 Einzelrechtsnachfolge. 461

2.3.3 Formwechsel. 462

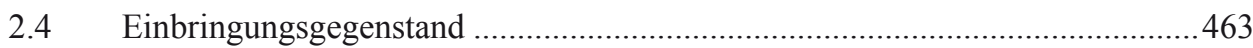

2.4.1 Betrieb und Teilbetrieb .................................................................................464

2.4.1.1 Übertragung der wesentlichen Betriebsgrundlagen.................................465

2.4.1.2 Zurückbehaltung wesentlicher Betriebsgrundlagen ................................467

2.4.1.3 Zurückbehaltung nicht wesentlicher Betriebsgrundlagen ......................470

2.4.2 Mitunternehmeranteil ..........................................................................4 470

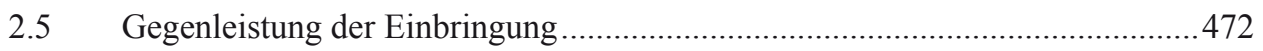

2.6 An der Einbringung beteiligte Personen............................................................ 474

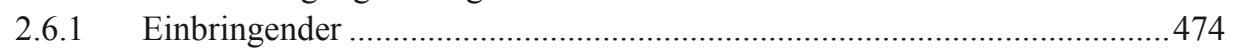

2.6.2 Übernehmende Kapitalgesellschaft ...........................................................477

2.7 Steuerliches Bewertungswahlrecht der Übernehmerin......................................478

2.7.1 Verhältnis des steuerlichen Bewertungswahlrechts zum Handelsrecht .......478

2.7.1.1 Handelsbilanzielle Aufstockungspflicht..............................................478

2.7.1.2 Ausgleichsposten.......................................................................... 481

2.7.1.3 Bilanzsteuerrechtliche Behandlung des Ausgleichspostens .....................483

2.7.2 Voraussetzungen für das Bewertungswahlrecht ..........................................484

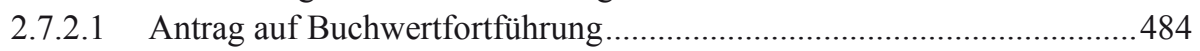

2.7.2.2 Zusätzliche Wirtschaftsgüter als Gegenleistung ....................................494

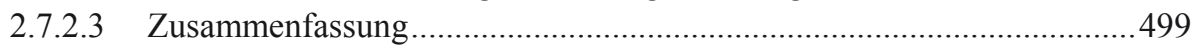




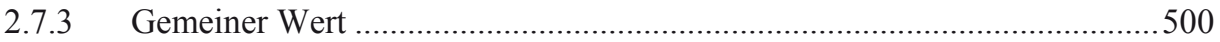

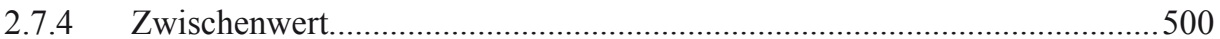

2.8 Veräußerungspreis und Ansatz der gewährten Gesellschaftsanteile ..................502

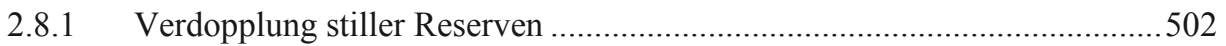

2.8.2 Veräußerungspreis des Betriebsvermögens .................................................504

2.8.3 Anschaffungskosten der Kapitalgesellschaftsanteile ...................................504

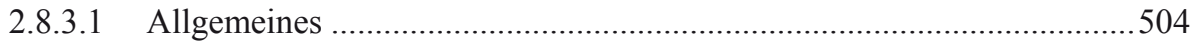

2.8.3.2 Die Auswirkung der Einbringung ausländischen

Betriebsstättenvermögens auf die Anschaffungskosten der

Kapitalgesellschaftsanteile ...................................................................506

2.8.3.3 Die Auswirkung der Gewährung zusätzlicher Wirtschaftsgüter auf die Anschaffungskosten der Kapitalgesellschaftsanteile........................508

2.9 Qualifikation des originären Einbringungsgewinns ........................................513

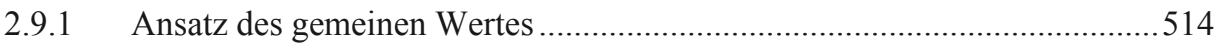

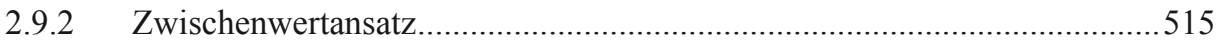

2.10 Anrechnung fiktiver ausländischer Steuern.....................................................516

2.11 Zeitpunkt der Einbringung und steuerliche Rückwirkung................................517

2.11.1 Rückbeziehung des steuerlichen Übertragungszeitpunkts ...........................517

2.11.2 Steuerlicher Übertragungsstichtag bei Gesamtrechtsnachfolge.....................518

2.11.3 Steuerlicher Übertragungsstichtag bei Einzelrechtsnachfolge.......................519

2.11.4 Versagung der Rückbeziehung bei doppelter Nichtbesteuerung ..................520

2.11.5 Vorfälle im Rückwirkungszeitraum.......................................................520

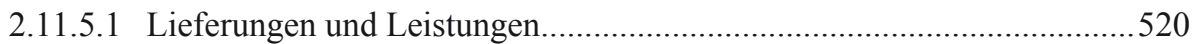

2.11.5.2 Entnahmen und Einlagen ..................................................................521

2.12 Besteuerung des Anteilseigners.................................................................521

2.12.1 Gefahr des Missbrauchs der steuerneutralen Einbringung............................521

2.12.2 Die rückwirkende Besteuerung der Einbringung ..........................................525

2.12.2.1 Systematik der Sperffristenregelung …………....................................55

2.12.2.2 Systematik der rückwirkenden Besteuerung ............................................528

2.12.2.3 Der Einbringungsgewinn I ..................................................................529

2.12.2.3.1 Ermittlung des Einbringungsgewinns I ............................................529

2.12.2.3.2 Schädliche Veräußerung eines Teils der gewährten Anteile .............534

2.12.2.3.3 Bei Betriebseinbringung mit eingebrachte Anteile............................534

2.12.2.4 Qualifikation des Einbringungsgewinns I .............................................535

2.12.2.5 Gefahr einer Doppelbesteuerung der stillen Reserven .............................536

2.12.2.6 Nachträgliche Anschaffungskosten beim Einbringenden........................538

2.12.2.7 Verhältnis von Einbringungsgewinn I und Veräußerungsgewinn.............539

2.12.2.8 Gefahr der Doppelbesteuerung bei der übernehmenden Gesellschaft.....543

2.12.3 Zusammenfassung der Systematik des Einbringungsgewinns......................547

2.12.4 Nachweispflichten des Einbringenden.....................................................549

2.12.5 Veräußerungsgleiche Ersatztatbestände.....................................................549

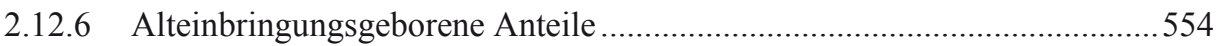

2.12.6.1 Veräußernder ist eine natürliche Person....................................................557

2.12.6.1.1 Begünstigung des Veräußerungsgewinns ........................................557 
2.12.6.1.2 Steuerbegünstigungen bei Veräußerung durch eine natürliche

Person.... .560

2.12.6.2 Veräußernder ist eine juristische Person .................................................560

2.13 Auswirkungen auf die übernehmende Gesellschaft ........................................566

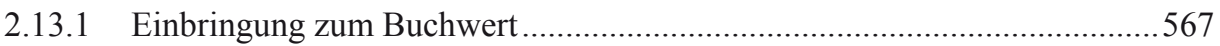

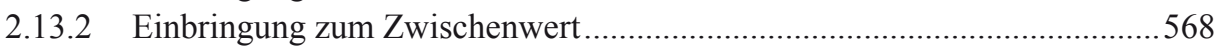

2.13.3 Einbringung zum gemeinen Wert ...........................................................5 570

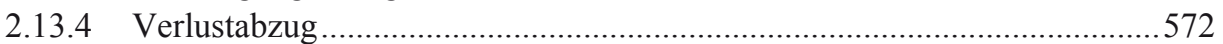

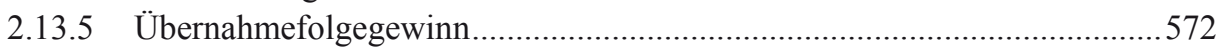

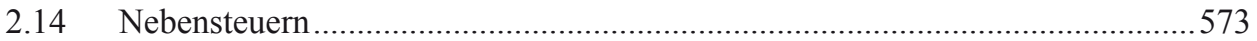

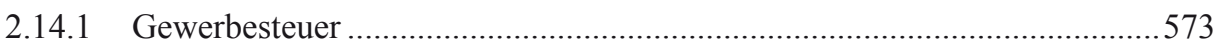

2.14.2 Grunderwerbsteuer ............................................................................ 573

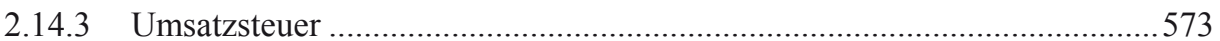

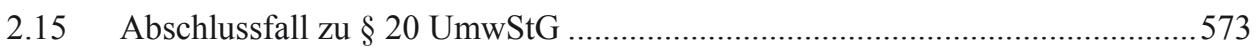

3 Anteilstausch gem. § 21 UmwStG ......................................................................579

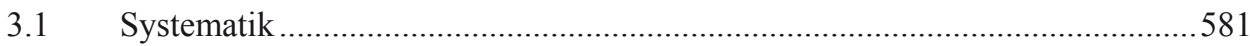

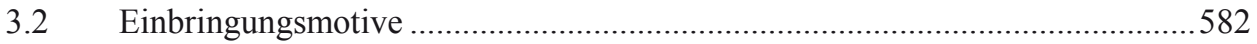

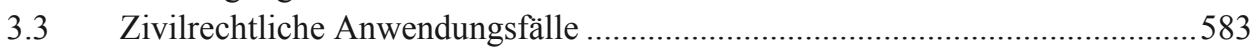

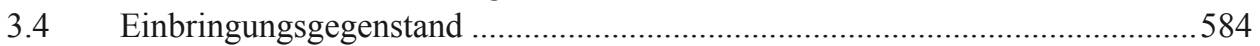

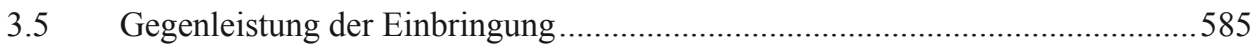

3.6 An der Einbringung beteiligte Personen........................................................586

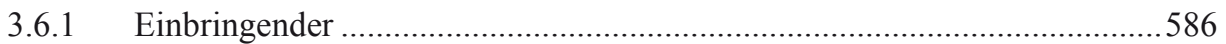

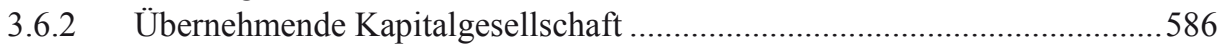

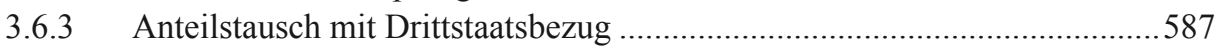

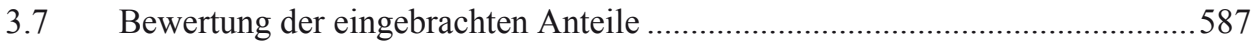

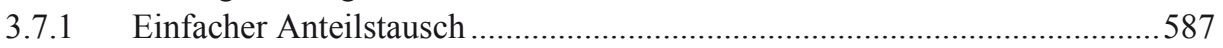

3.7.2 Bewertungswahlrecht bei qualifiziertem Anteilstausch............................58

3.7.2.1 Antrag auf Buchwertfortführung.........................................................58

3.7.2.2 Zusätzliche Wirtschaftsgüter als Gegenleistung ...................................590

3.8 Veräußerungspreis und Ansatz der gewährten Gesellschaftsanteile .................591

3.8.1 Grundsatz der doppelten Wertverknüpfung ...............................................591

3.8.2 Ausnahme von der Wertverknüpfung bei grenzüberschreitendem

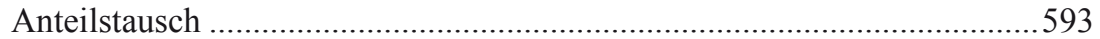

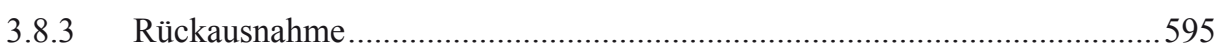

3.8.4 Auswirkung von zusätzlichen Gegenleistungen auf die

Anschaffungskosten der erhaltenen Anteile und Fortgeltung alteinbringungsgeborener Anteile...........................................................596

3.9 Qualifikation des originären Einbringungsgewinns ....................................597

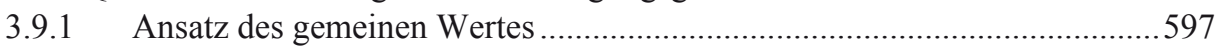

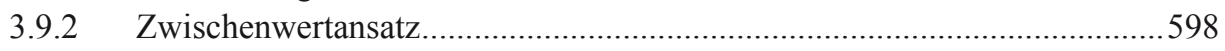

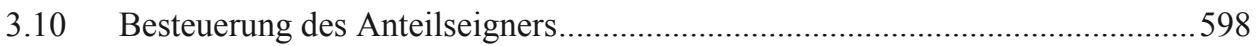

3.10.1 Gefahr des Missbrauchs der steuerneutralen Einbringung..........................598 
3.10.2 Die rückwirkende Besteuerung der Einbringung......................................601

3.10.2.1 Entstehung von Einbringungsgewinn II ..............................................601

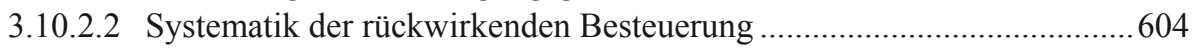

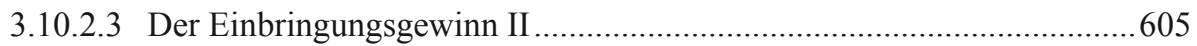

3.10.2.4 Qualifikation des Einbringungsgewinns II .............................................606

3.10.2.5 Gefahr einer Doppelbesteuerung der stillen Reserven ...........................607

3.10.2.5.1 Doppelbesteuerung beim Einbringenden.......................................607

3.10.2.5.2 Doppelbesteuerung bei der übernehmenden Gesellschaft ................609

3.10.3 Zusammenfassung der Systematik des Einbringungsgewinns......................610

3.10.4 Nachweispflichten des Einbringenden.......................................................612

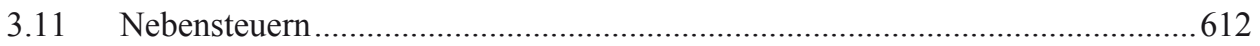

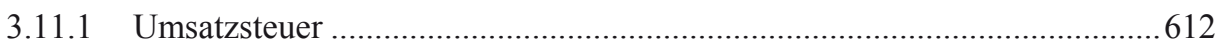

3.12 Vergleich Einbringungsgewinn I mit Einbringungsgewinn II..........................613

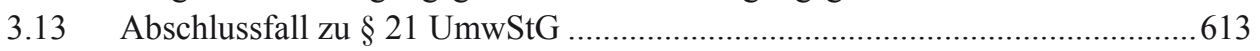

4 Einbringung in eine Personengesellschaft gem. $\$ 24$ UmwStG ............................617

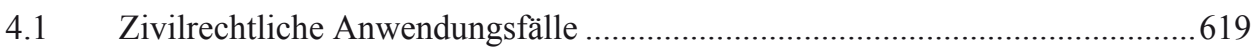

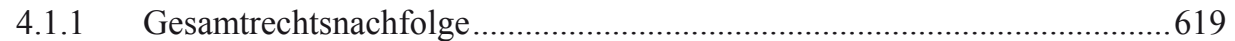

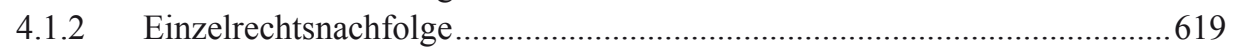

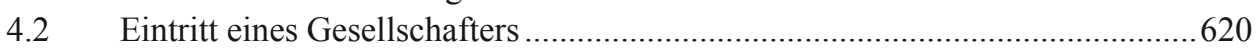

4.3 An der Einbringung beteiligte Personen.........................................................621

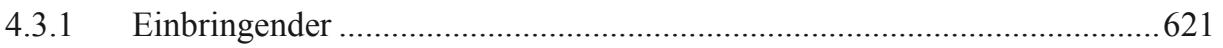

4.3.2 Übernehmende Personengesellschaft........................................................622

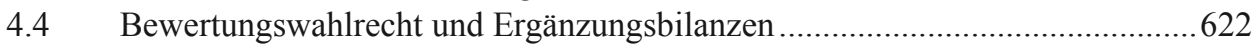

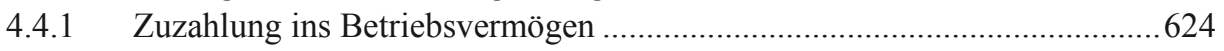

4.4.2 Zuzahlung ins Privatvermögen ..............................................................628

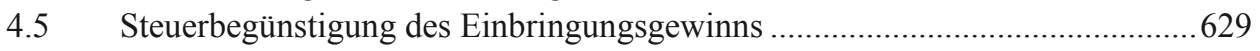

4.6 Nachträgliche Besteuerung bei Einbringung von Anteilen an

4.7 Analoge Anwendung der Regelungen zu $\S \S 20,23$ UmwStG ..........................632

4.7.1 Auswirkungen bei der übernehmenden Personengesellschaft ......................632

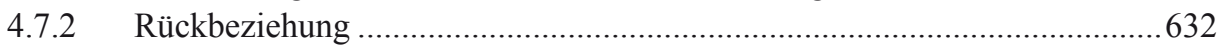

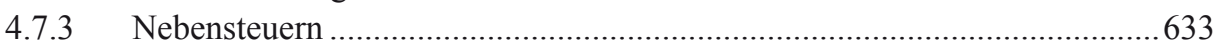

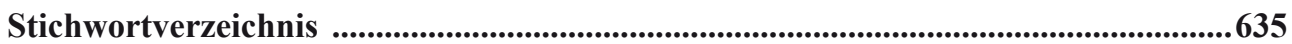




\section{Abbildungsverzeichnis}

Abbildung 1: Gesellschaftsrechtliche Unterschiede zwischen Kapital- und

Personengesellschaften

Abbildung 2: $\quad$ Steuerrechtliche Unterschiede zwischen Kapital- und

Personengesellschaften

Abbildung 3: Gewinnausschüttungen und Veräußerungsgewinne im Vergleich ............7

Abbildung 4: Behandlung der nichtabzugsfähigen Betriebsausgaben ............................ 12

Abbildung 5: Ermittlung des thesaurierungsfähigen Gewinns .................................... 13

Abbildung 6: Steuerbelastung von Kapitalgesellschaften im Vergleich zu

Personenunternehmen ohne Thesaurierungsbegünstigung...................... 14

Abbildung 7: $\quad$ Steuerbelastung von Kapitalgesellschaften im Vergleich zu

Personenunternehmen mit Thesaurierungsbegünstigung ....................... 15

Abbildung 8: Berücksichtigung der Gewerbesteuer bei Kapitalgesellschaften und

Personenunternehmen im Vergleich ................................................... 17

Abbildung 9: $\quad$ Umwandlungsarten nach dem UmwG ...................................................... 19

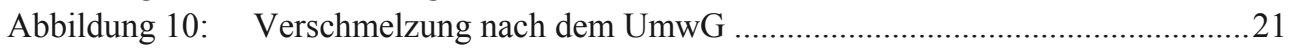

Abbildung 11: Verschmelzungsfähige Rechtsträger .......................................................21

Abbildung 12: Relevante Verschmelzungsmöglichkeiten nach dem UmwG ..................22

Abbildung 13: Spaltungsarten nach dem Umwandlungsgesetz ....................................23

Abbildung 14: Spaltungsfähige Rechtsträger..........................................................24

Abbildung 15: Relevante Auf- und Abspaltungsmöglichkeiten nach dem UmwG..........24

Abbildung 16: Relevante Ausgliederungsmöglichkeiten nach dem UmwG...................24

Abbildung 17: Aufspaltung nach dem UmwG.............................................................25

Abbildung 18: Vergleich zwischen der Verschmelzung durch Neugründung und der

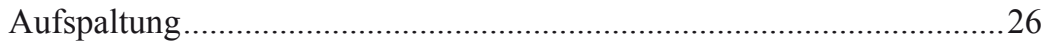

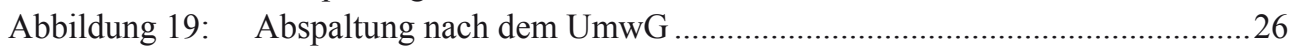

Abbildung 20: Ausgliederung nach dem UmwG .....................................................2

Abbildung 21: Unterschiede zwischen den Spaltungsarten ...........................................28

Abbildung 22: In den Formwechsel nach dem UmwG einbezogene Rechtsträger ..........29

Abbildung 23: Relevante Formwechsel nach dem UmwG ........................................29

Abbildung 24: Aufbau des Umwandlungssteuergesetzes ........................................... 31

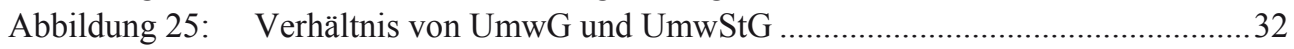

Abbildung 26: Verschmelzung von Kapitalgesellschaft(en) auf eine

Personengesellschaft ......................................................................... 34

Abbildung 27: Verschmelzungsfähige Rechtsträger (KapGes auf PersGes) ...................36

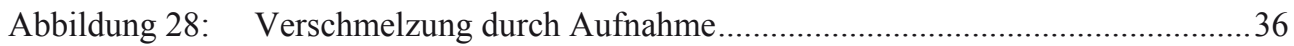

Abbildung 29: Verschmelzung durch Neugründung .................................................. 37

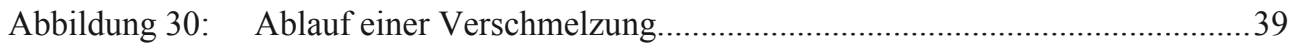

Abbildung 31: Inhalt des Verschmelzungsvertrags bei der Verschmelzung einer

Kapitalgesellschaft auf eine Personengesellschaft .................................40

Abbildung 32: Gewährung von Gesellschaftsrechten bei der Verschmelzung

KapGes auf PersGes im Überblick 
Abbildung 33: Handelsrechtliche Schlussbilanz und Verschmelzungsstichtag ... 47

Abbildung 34: Grenzüberschreitende Verschmelzung KapGes auf PersGes....

Abbildung 35: Handelsrechtlicher Verschmelzungsgewinn oder -verlust bei

Buchwertfortführung.

Abbildung 36: Regelungen zur Verschmelzung KapGes auf PersGes nach dem

UmwStG .................................................................................5 55

Abbildung 37: Realisation und Besteuerung von stillen Reserven ................................57

Abbildung 38: Möglichkeit der Buchwertfortführung ...................................................58

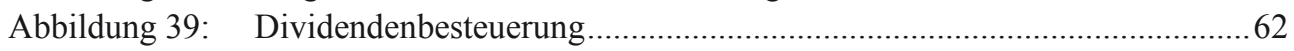

Abbildung 40: Transparenz- und Intransparenzprinzip bei der Besteuerung von

Kapital- und Personengesellschaften ....................................................63

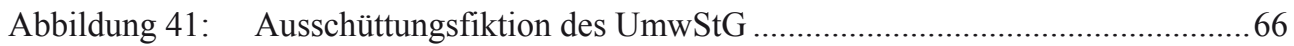

Abbildung 42: Steuerverstrickung bei der Verschmelzung von KapGes auf PersGes .....67

Abbildung 43: Wahl des handelsrechtlichen Umwandlungsstichtags und

Auswirkung auf die Besteuerung .........................................................6 69

Abbildung 44: Steuerlicher Rückwirkungszeitraum ...................................................... 70

Abbildung 45: Ausscheiden von Anteilseignern ................................................................ 71

Abbildung 46: Steuerliche Behandlung beim Ausscheiden von Anteilseignern .............. 72

Abbildung 47: Gewinnausschüttungen während des Rückwirkungszeitraums .................73

Abbildung 48: Behandlung von Gewinnausschüttungen im Rückwirkungszeitraum bei der Verschmelzung KapGes auf PersGes.........................................75

Abbildung 49: Zahlungen an Gesellschafter der übertragenden Kapitalgesellschaft.......77

Abbildung 50: Voraussetzung des Bewertungswahlrechts des $\S 3$ Abs. 2 UmwStG....... 85

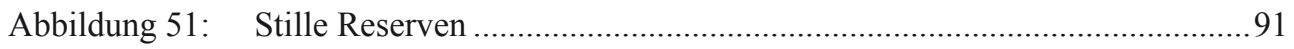

Abbildung 52: Auswirkungen bei Aufstockung der Buchwerte .................................... 92

Abbildung 53: Ermittlung des Übertragungsergebnisses ..............................................93

Abbildung 54: Auswirkung des Bewertungswahlrechts von $\S 3$ UmwStG .....................94

Abbildung 55: $\quad$ Übertragung eines Verlustvortrages........................................................ 98

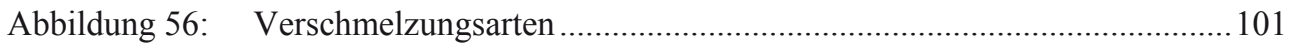

Abbildung 57: Möglichkeiten zur Vermeidung einer Doppelbesteuerung....................... 103

Abbildung 58: Inlandsverschmelzung mit Auslandsbezug, DBA-

Freistellungsmethode

Abbildung 59: Inlandsverschmelzung mit Auslandsbezug, DBA-

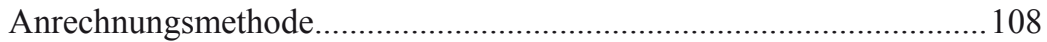

Abbildung 60: Ansatz der Aktiva in der steuerlichen Schlussbilanz bei einer

Inlandsverschmelzung mit Auslandsbezug ........................................ 110

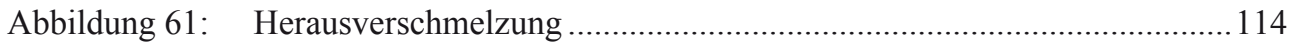

Abbildung 62: Auslandsverschmelzung mit Inlandsbezug ....................................... 116

Abbildung 63: Hineinverschmelzung........................................................................ 117

Abbildung 64: Steuerliche Schlussbilanz und steuerliche Übernahmebilanz ................ 119

Abbildung 65: Wertverknüpfung (§ 4 Abs. 1 Satz 1 UmwStG) ................................... 119

Abbildung 66: Beteiligungskorrekturgewinn auf Ebene der übernehmenden

PersGes ................................................................................... 121

Abbildung 67: Ermittlung des steuerlichen Übernahmeergebnisses .............................. 126 
Abbildung 68: Berechnung des Übernahmeergebnisses 130

Abbildung 69: Zeitpunkte des Erwerbs der Anteile an der KapGes ......... .131

Abbildung 70:

Zweck des $§ 5$ UmwStG 135

Abbildung 71:

Fälle der Anschaffungs-/ Einlagefiktion des $\S 5$ UmwStG

Abbildung 72:

Grund für die unterschiedliche Behandlung bei der Einlagefiktion

Abbildung 73:

Einbeziehung von Anteilen in die Ermittlung des

Übernahmeergebnisses.

Abbildung 74: Steuerliche Behandlung eines Übernahmeverlustes...

Abbildung 75:

1,6-fache Besteuerung der stillen Reserven aufgrund der

Nichtberücksichtigung eines Übernahmeverlusts

Abbildung 76:

Steuerliche Behandlung des Übernahmegewinns

Abbildung 77:

Sinn und Zweck des $\S 18$ Abs. 3 UmwStG.

Abbildung 78:

Steuerliche Behandlung des Übernahmeergebnisses

Abbildung 79:

Vergleich von Übertragungs- und Übernahmegewinn

Abbildung 80:

Zusammenspiel von Übertragungsgewinn und Bezüge i.S.d. § 7

UmwStG

Abbildung 81: Grunderwerbsteuerbefreiung des $\S 6 \mathrm{a} \mathrm{GrEStG..}$

Abbildung 82: Formwechsel unter Beteiligung von Kapital- und

Personengesellschaften ....

Abbildung 83: Verschmelzung von Kapitalgesellschaften ....

Abbildung 84: Steuerliche Behandlung der Verschmelzung von

Kapitalgesellschaften auf eine Personengesellschaft bzw. auf eine

Kapitalgesellschaft

Abbildung 85: Unterschied und Gemeinsamkeiten Verschmelzung KapPers und

KapKap

Abbildung 86: Inhalt des Verschmelzungsplans gem. § 122c UmwG.....

Abbildung 87: Handelsrechtlicher Verschmelzungsgewinn oder -verlust bei

Buchwertfortführung.

Abbildung 88:

Grundfälle der Verschmelzung

Abbildung 89:

Anwendungsbereich der $\S \S 11-13 \mathrm{UmwStG}$

Abbildung 90:

Die Entstehung weißer Einkünfte bei Verschmelzungen.

Abbildung 91:

Verschmelzungsvarianten .

Abbildung 92:

„Up-stream merger“"

Abbildung 93:

„Down-stream merger“

Abbildung 94:

Das Bewertungswahlrecht gem. $§ 11$ Abs. 2 Satz 1 UmwStG.

Abbildung 95: Gegenüberstellung von handels- und steuerrechtlichen

Bewertungsvorschriften für die Schlussbilanz der übertragenden

Kapitalgesellschaft.

Abbildung 96: Ausschluss des Besteuerungsrechts - Situation vor der

Verschmelzung

Abbildung 97: Ausschluss des Besteuerungsrechts. Situation nach der

Verschmelzung ....

Abbildung 98: Ausschluss und Beschränkung des deutschen Besteuerungsrechts . 
Abbildung 99: Die Anwendung des Bewertungswahlrechts gem. § 11 Abs. 2

UmwStG bei inländischen Verschmelzungen....

Abbildung 100: Die Anwendung des Bewertungswahlrechts nach $\S 11$ Abs. 2

UmwStG bei Herausverschmelzungen.

Abbildung 101: Entstehung eines Übertragungsgewinns.

.245

Abbildung 102: Wertverknüpfung gem. § 12 Abs. 1 Satz 1 UmwStG ............................250

Abbildung 103: Auswirkung der Aufstockung der Buchwerte .......................................251

Abbildung 104: Übernahmegewinn entspricht Gewinnrücklagen ..................................254

Abbildung 105: Vergleich des Übernahmegewinns von Kap/Pers zu Kap/Kap ..............255

Abbildung 106: Auswirkungen bei Aufstockung der Buchwerte ...................................256

Abbildung 107: Vergleich der Besteuerung des Übernahmegewinns von Kap/Pers zu

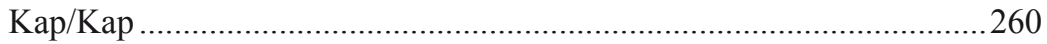

Abbildung 108: Wertaufholungsgebot bei down-stream und up-stream merger im

Vergleich...............................................................................2. 265

Abbildung 109: Behandlung von Verlustvorträgen ........................................................267

Abbildung 110: Zweistufiger Untergang von Verlustvorträgen....................................268

Abbildung 111: Untergang des Verlustvortrages durch Erwerb und Verschmelzung .....269

Abbildung 112: Anwendung von § 8c KStG im Rahmen von Verschmelzungen ...........271

Abbildung 113: Stille Reserven Regel des $\S 8 \mathrm{c}$ Abs. 1 Satz 6-8 KStG .............................2 274

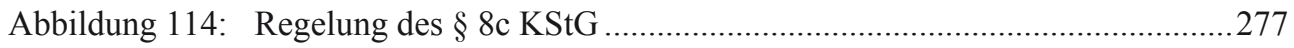

Abbildung 115: Verwendungsreihenfolge für die Finanzierung einer

Gewinnausschüttung ...............................................................28

Abbildung 116: Minderung des Sonderausweises durch das steuerliche Einlagekonto...292

Abbildung 117: Das Bewertungswahlrecht gem. § 13 Abs. 2 Satz 1 UmwStG...............313

Abbildung 118: Buchwertansatz .................................................................................... 314

Abbildung 119: Kein Ausschluss des Besteuerungsrechts; Situation vor Verschmelzung ............................................................................... 316

Abbildung 120: Kein Ausschluss des Besteuerungsrechts; Situation nach

Verschmelzung

Abbildung 121: Beschränkung des Besteuerungsrechts; Situation vor Verschmelzung .. 318

Abbildung 122: Beschränkung des Besteuerungsrechts; Situation nach Verschmelzung

Abbildung 123: Art der Anteile der Gesellschafter der übertragenden

Kapitalgesellschaft

Abbildung 124: Gewerbesteuerliche Folgen der Verschmelzung von

Kapitalgesellschaften

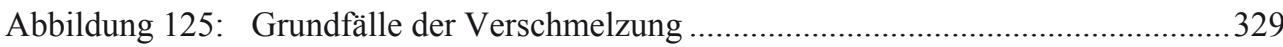

Abbildung 126: Inlandsverschmelzung mit Betriebsvermögen im EU-Ausland ..............332

Abbildung 127: Innerstaatliche Auslandsverschmelzung mit dt. Betriebsvermögen........333

Abbildung 128: Innerstaatliche Auslandsverschmelzung mit dt. Anteilseigner.................334

Abbildung 129: Grenzüberschreitende Auslandsverschmelzung mit Inlandsbezug ........334

Abbildung 130: Herausverschmelzung mit Zuordnung des deutschen

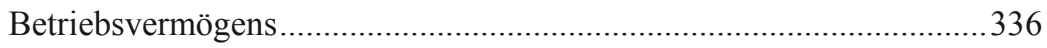

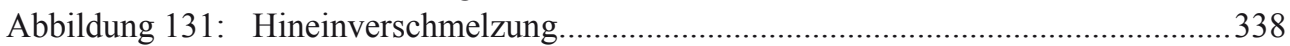


Abbildung 132: Spaltungsarten nach $\S 123$ UmwG. 348

Abbildung 133: Aufspaltung einer Kapitalgesellschaft 349

Abbildung 134: Abspaltung bei einer Kapitalgesellschaft

Abbildung 135: Ausgliederung bei einer Kapitalgesellschaft ........................................ 350

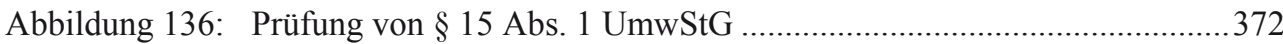

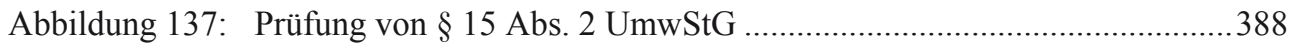

Abbildung 138: Das Bewertungswahlrecht gem. $§ 11$ Abs. 2 UmwStG...........................389

Abbildung 139: Verlustvortragsaufteilung bei Abspaltung .............................................406

Abbildung 140: Auswirkungen einer Buchwertaufstockung auf den Verlustvortrag bei Abspaltung

Abbildung 141: Unterschiede zwischen Aufspaltung und Abspaltung bei der Aufteilung der Eigenkapitalausweisbestände

Abbildung 142: Maßgebende Bestände und zeitliche Erfassung der jeweiligen Änderungen der steuerlichen Einlagekonten.

Abbildung 143: Das Bewertungswahlrecht gem. § 13 Abs. 2 Satz 1 UmwStG. 419

Abbildung 144: Gesamtübersicht über die steuerliche Behandlung der Spaltung von Kapitalgesellschaften I

Abbildung 145: Gesamtübersicht über die steuerliche Behandlung der Spaltung von Kapitalgesellschaften II.

Abbildung 146: Zivilrechtliche Anwendungsfälle umwandlungssteuerrechtlicher Tatbestände

Abbildung 147: Aufdeckung und steuerliche Erfassung stiller Reserven durch Veräußerung.

Abbildung 148: Aufdeckung und steuerliche Erfassung stiller Reserven durch Tausch

Abbildung 149: Anwendung der Einbringungsvorschriften in Abhängigkeit vom Gegenstand der Einbringung

Abbildung 150: Anwendung der Einbringungsvorschriften in Abhängigkeit von der Gegenleistung

Abbildung 151: Prinzipien bei Betriebsveräußerung bzw. bei Einbringung...

Abbildung 152: Buchwertfortführung und spätere Entstrickung von stillen Reserven.... 446

Abbildung 153: Beteiligungsstrukturen bei Verschmelzung bzw. Einbringung ...............447

Abbildung 154: Empfänger der Gegenleistung.............................................................. 450

Abbildung 155: Erfassung der Gewinnrücklagen bei Übertragung von Vermögen einer Kapitalgesellschaft.

Abbildung 156: Bewertungswahlrecht des übernehmenden Rechtsträgers bei Einbringung.....

Abbildung 157: Stille Reserven im Einbringungskreislauf.

Abbildung 158: Außerkraftsetzung der Rechtsfolge des $§ 16 \mathrm{EStG}$ durch $\S 20$ UmwStG 456

Abbildung 159: Regelungen zur Einbringung von Vermögen in eine Kapitalgesellschaft.... .458

Abbildung 160: Schicksal des Überträgers und Empfänger der Gegenleistung. 461 
Abbildung 161: Zivilrechtliche Anwendungsfälle im Rahmen einer Einbringung in eine Kapitalgesellschaft 463

Abbildung 162: Unterschiedliche Erfassung von wesentlichen Betriebsgrundlagen ....... 466

Abbildung 163: Tausch bei Zurückbehaltung wesentlicher Betriebsgrundlagen .............468

Abbildung 164: Aufgabegewinn bei Zurückbehaltung wesentlicher Betriebsgrundlagen

Abbildung 165: Sonderbetriebsvermögen I als wesentliche Betriebsgrundlage 472

Abbildung 166: Kollision zwischen handelsrechtlicher Zwangswertaufstockung und steuerlicher Buchwertfortführung

Abbildung 167: Ausgleichsposten bei handelsrechtlicher Höherbewertung..... 482

Abbildung 168: Aus negativem Vermögen resultieren negative Anschaffungskosten .... 486

Abbildung 169: Zwangswertaufstockung zur Vermeidung negativer Anschaffungskosten

Abbildung 170:

Einschränkung des deutschen Besteuerungsrechts.

Abbildung 171: Einbringung ausländischer Betriebsstätte in ausländische Gesellschaft.

Abbildung 172: Einbringung deutscher Betriebsstätte in ausländische Gesellschaft.......493

Abbildung 173: Gewährung zusätzlicher Gegenleistungen

Abbildung 174:

Zusätzliche Gegenleistungen über dem gemeinen Wert des

Betriebsvermögens

Abbildung 175:

Einschränkungen des steuerlichen Bewertungswahlrechts

Abbildung 176:

Verdopplung stiller Reserven.

Abbildung 177:

Äquivalenzfunktion der gewährten Kapitalgesellschaftsanteile.

.505

Abbildung 178:

Übertragung stiller Reserven in Folge einer Gewährung anderer

Wirtschaftsgüter

Abbildung 179:

Sicherstellung der steuerlichen Erfassung stiller Reserven...

Abbildung 180:

Sicherstellung der Erfassung stiller Reserven durch

Anschaffungskosten von Null

Abbildung 181: Besteuerung des Einbringungsgewinns bei Ansatz des gemeinen

Wertes .....

Abbildung 182: Steuerlich maßgeblicher Übertragungszeitpunkt .....

Abbildung 183:

Steuerlicher Rückwirkungszeitraum bei Gesamtrechtsnachfolge .

Abbildung 184:

Steuerlicher Rückwirkungszeitraum bei Einzelrechtsnachfolge .

Abbildung 186:

Tausch Betriebsvermögen gegen Anteile als Steuersparmodell?...

Abbildung 187:

Allgemeine Veräußerungsprivilegien für Kapitalgesellschaftsanteile ...524

Abbildung 188:

Veräußerung und schädliche Veräußerung

.526

Abbildung 189:

Abbildung 190:

Schädliche Veräußerung innerhalb der Sperrfrist .526

Rückwirkung bei schädlicher Veräußerung innerhalb der Sperrfrist .....528

Abbildung 191:

Bemessungsgrundlage des Einbringungsgewinns I .531

Abbildung 192:

Abbildung 193:

Verringerung des Einbringungsgewinns I über den Zeitablauf..

Berechnung des Einbringungsgewinns I

Entstehung des Einbringungsgewinns I

Abbildung 194:

EBG I als nachträgliche Anschaffungskosten des Einbringenden .

Abbildung 195: Schädliche Veräußerung nach 4 Jahren 
Abbildung 196: Besteuerung der stillen Reserven .......................................................542

Abbildung 197: EBG I als Erhöhungsbetrag bei der übernehmenden Gesellschaft.........545

Abbildung 198: EBG I als Abschreibungspotenzial bei der übernehmenden

Gesellschaft .546

Abbildung 199: Vermeidung der Doppelbesteuerung durch den Einbringungsgewinn

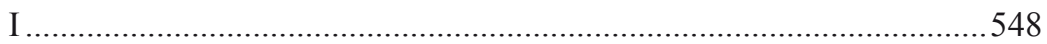

Abbildung 200: Entstehung einbringungsgeborener Anteile i.S.d. UmwStG a.F...........556

Abbildung 201: Versagung der Begünstigungen des Halbeinkünfteverfahrens für einbringungsgeborene Anteile i.S.d. § $21 \mathrm{UmwStG}$ a.F.

Abbildung 202: Einbringung und Veräußerung der Anteile durch eine Kapitalgesellschaft .561

Abbildung 203: Vergleich wesentlicher Merkmale der Missbrauchsregelungen..............566

Abbildung 204: Einbringung von Anteilen in eine Kapitalgesellschaft............................580

Abbildung 205: Regelungen zur Einbringung von Anteilen in eine

Kapitalgesellschaft

Abbildung 206: Status der als Gegenleistung gewährten Anteile ....................................585

Abbildung 207: Begründung und Aufstockung einer Mehrheitsbeteiligung ...................589

Abbildung 208: Einbringung von Anteilen in eine Kapitalgesellschaft...........................592

Abbildung 209: Besteuerung des Einbringungsgewinns bei Ansatz des gemeinen

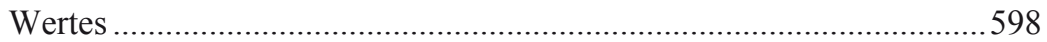

Abbildung 210: Missbrauchsrisiko bei Veräußerungsvorgängen nach Anteilstausch ......599

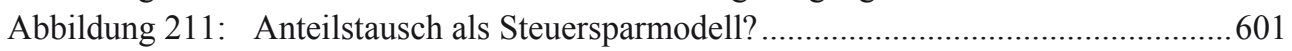

Abbildung 212: Schädliche Veräußerung als Auslöser des Einbringungsgewinns...........604

Abbildung 213: Auseinanderfallen von Veräußerndem und Träger des EBG II .............605

Abbildung 214: Berechnung des Einbringungsgewinns II.................................................606

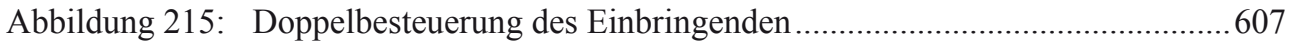

Abbildung 216: Vermeidung der Doppelbesteuerung durch den Einbringungsgewinn

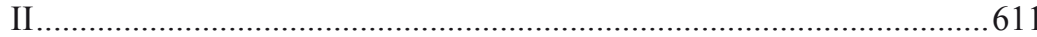

Abbildung 217: Einbringung von Betriebsvermögen in eine Personengesellschaft.........618

Abbildung 218: Entstehung einer neuen Personengesellschaft aus Sicht des $§ 24$

UmwStG

Abbildung 219: Eintritt und Wechsel in eine Personengesellschaft...............................621

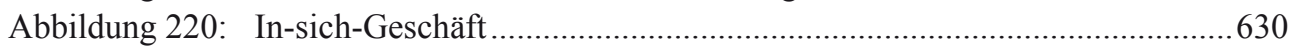




\section{Abkürzungs- und Symbolverzeichnis}

a.A.

Abs.

a.F.

AfA

AG

AHK

AktG

AO

AV

BB

$\mathrm{b} \& \mathrm{~b}$

BewG

$\mathrm{BFH}$

BGB

BMG

Bst.

BStB1.

BT

BR-Drucks.

BT-Drucks.

DB

DStR

DStZ

EFG

EK

ErbSt

ErbStG

ESt

EStG

EStR

EuGH

EWIV

FG

FK

FN-IDW

FR

FRL

GA

GbR

gem.

GewSt andere(r) Ansicht

Absatz

alte Fassung

Abschreibung

Aktiengesellschaft

Anschaffungs- oder Herstellungskosten

Aktiengesetz

Abgabenordnung

Anlagevermögen

Betriebs-Berater (Zeitschrift)

Bilanz \& Buchhaltung (Zeitschrift)

Bewertungsgesetz

Bundesfinanzhof

Bürgerliches Gesetzbuch

Bemessungsgrundlage

Buchstabe

Bundessteuerblatt

Bundestag

Bundesrats-Drucksache

Bundestags-Drucksache

Der Betrieb (Zeitschrift)

Deutsches Steuerrecht (Zeitschrift)

Deutsche Steuerzeitung (Zeitschrift)

Entscheidungen der Finanzgerichte (Zeitschrift)

Eigenkapital

Erbschaftsteuer

Erbschaftsteuergesetz

Einkommensteuer

Einkommensteuergesetz

Einkommensteuerrichtlinien

Europäischer Gerichtshof

Europäische Wirtschaftliche Interessenvereinigung

Finanzgericht

Fremdkapital

IDW-Fachnachrichten (Zeitschrift)

Finanz-Rundschau (Zeitschrift)

Fusionsrichtlinie

Gewinnausschüttung

Gesellschaft bürgerlichen Rechts

gemäß

Gewerbesteuer 


\begin{tabular}{|c|c|}
\hline GewStG & Gewerbesteuergesetz \\
\hline GewStR & Gewerbesteuerrichtlinien \\
\hline GFW & Geschäfts- oder Firmenwert \\
\hline ggf. & gegebenenfalls \\
\hline $\mathrm{GmbH}$ & Gesellschaft mit beschränkter Haftung \\
\hline GmbHG & GmbH-Gesetz \\
\hline GmbHR & GmbH-Rundschau (Zeitschrift) \\
\hline GoB & Grundsätze ordnungsmäßiger Buchführung \\
\hline GrESt & Grunderwerbsteuer \\
\hline GrEStG & Grunderwerbsteuergesetz \\
\hline HFA & Hauptfachausschuss \\
\hline HGB & Handelsgesetzbuch \\
\hline h.M. & herrschende(r) Meinung \\
\hline hr. & handelsrechtlich \\
\hline Hrsg. & Herausgeber \\
\hline HS. & Halbsatz \\
\hline IDW & Institut der Wirtschaftsprüfer \\
\hline IRC & Internal Revenue Code \\
\hline IStR & Internationales Steuerrecht (Zeitschrift) \\
\hline KapGes & Kapitalgesellschaft \\
\hline KG & Kommanditgesellschaft \\
\hline $\mathrm{KGaA}$ & Kommanditgesellschaft auf Aktien \\
\hline $\mathrm{KSt}$ & Körperschaftsteuer \\
\hline KStG & Körperschaftsteuergesetz \\
\hline LG & Landgericht \\
\hline Ltd. & Limited (englische Aktiengesellschaft) \\
\hline NK & Nominalkapital \\
\hline Nr. & Nummer \\
\hline n.V. & nach Verschmelzung \\
\hline OHG & offene Handelsgesellschaft \\
\hline OLG & Oberlandesgericht \\
\hline p.a. & per annum \\
\hline pers. & persönlich \\
\hline PersGes & Personengesellschaft \\
\hline PV & Privatvermögen \\
\hline Rz. & Randziffer \\
\hline S.A. & $\begin{array}{l}\text { Sociedad Anónima (spanische Aktiengesellschaft)/ } \\
\text { Société Anonyme (französische Aktiengesellschaft) }\end{array}$ \\
\hline SE & Societas Europaea \\
\hline SE-VO & Verordnung über das Statut der SE \\
\hline S.P.A. & Società per azioni (italienische Aktiengesellschaft) \\
\hline StB & Der Steuerberater (Zeitschrift) \\
\hline StBereinG & Steuerbereinigungsgesetz \\
\hline SteuerStud & Steuer und Studium (Zeitschrift) \\
\hline
\end{tabular}


StSenkG

StuW

StVergAbG

Tz.

ÜN

ÜT

UmwBerG

UmwG

UmwStE

UmwStG

UnStFG

USt

UStG

UV

vEK

VSt

v.V.

$\mathrm{VZ}$

Wpg
Steuersenkungsgesetz

Steuer und Wirtschaft (Zeitschrift)

Steuervergünstigungsabbaugesetz

Textziffer

Übernehmerin

Überträgerin

Gesetz zur Bereinigung des Umwandlungsrechts

Umwandlungsgesetz

Umwandlungssteuererlass

Umwandlungssteuergesetz

Unternehmensteuerfortentwicklungsgesetz

Umsatzsteuer

Umsatzsteuergesetz

Umlaufvermögen

verwendbares Eigenkapital

Vermögensteuer

vor Verschmelzung

Veranlagungszeitraum

Die Wirtschaftsprüfung (Zeitschrift) 